\title{
Primary chemoprevention of breast cancer: Are the adverse effects too burdensome?
}

\author{
Vinay Prasad MD MPH, Marie Diener-West PhD
}

Competing interests: None declared.

This article has been peer reviewed.

Correspondence to: Vinay Prasad, vinayak.k. prasad@gmail.com

CMAJ 2015. DOI:10.1503 /cmaj.141627
$\mathrm{E}$ nthusiasm for the chemoprevention of breast cancer has grown in recent years. In 2013, an updated guideline from the US Preventive Services Task Force endorsed the use of tamoxifen and raloxifene in women at increased risk of breast cancer (grade B recommendation). ${ }^{1}$ Specifically, the task force concluded that the benefits of tamoxifen outweigh the risks among women aged 50-59 whose estimated five-year risk of invasive breast cancer is $4.5 \%$ or greater and that the net benefits of raloxifene occur when the estimated five-year risk exceeds $2 \%, 3 \%$ and $4 \%$ among women in their 50 s, 60 s and 70 s, respectively. ${ }^{1}$

This position is narrower than that of the American Society of Clinical Oncology, ${ }^{2}$ whose guideline advises women with an estimated fiveyear risk of breast cancer greater than $1.67 \%$ to discuss the use of tamoxifen, raloxifene and exemestane with their doctor. A Canadian guideline, ${ }^{3}$ although slightly older, similarly advises women whose five-year risk is greater than $1.67 \%$ to be counselled about the potential benefits of chemoprevention. The Canadian Cancer Society concurs, advising women at high risk of breast cancer to consider tamoxifen. ${ }^{4}$ In January 2014, the US Department of Health and Human Services announced that tamoxifen and raloxifene would be covered by insurance plans without copayment.

Collectively, these steps aim to increase the use of medications that have long been known

\section{- KEY POINTS}

- The benefits of chemoprevention of breast cancer are overestimated, and the harms are underappreciated.

- Trial data have not shown that chemoprevention of breast cancer reduces breast cancer mortality.

- Chemoprevention decreases incident breast cancer, but the effect is likely overestimated based on high rates of mammography and overdiagnosis.

- Convincing evidence should underpin any recommendation for a healthy patient to take potentially harmful preventive medication.

- The bar for primary chemoprevention of breast cancer should be set higher.

to decrease incident breast cancer but have been consistently underused in clinical practice. Renewed commitment to chemoprevention has the potential to affect many Canadian women. Estimates suggest that as many as $15 \%$ of women aged 35-79 years may be eligible for tamoxifen chemoprevention. ${ }^{5}$ Despite renewed interest in these drugs, questions remain. What is the effect of these drugs on mortality? Do the benefits justify the harms, particularly the burden of adverse effects?

Meta-analyses have confirmed that tamoxifen, raloxifene and other selective estrogenreceptor modulators reduce the rate of incident breast cancer. ${ }^{6}$ Randomized controlled trials (RCTs) have extended these findings to the aromatase inhibitors exemestane ${ }^{7}$ and anastrozole. ${ }^{6}$ The magnitude of this benefit is modest, with a number needed to treat of 40-60 women to prevent 1 case of incident breast cancer. ${ }^{6}$ In addition, chemoprevention offers mixed success among women at higzhest risk of breast cancer: those with germ-line BRCA1 and BRCA2 mutations. Although tamoxifen appears to reduce the incidence of cancer among women with $B R C A 2$ mutations, no effect has been found among those with $B R C A 1$ mutations. ${ }^{8}$

Although chemoprevention of breast cancer has been shown to decrease incident cancer, it has not been shown to avert breast cancer deaths. No drug has been found to reduce breast cancerspecific mortality or overall mortality in individual or pooled analyses. ${ }^{1,2,9}$ Some attribute this to insufficient follow-up, but chemoprevention trials collectively have more than 300000 person-years of follow-up. ${ }^{6}$ In addition, extended follow-up data (median $16 \mathrm{yr}$ ) from the International Breast Cancer Intervention Study I (IBIS-I) continue to show no difference in the number of deaths from breast cancer between the tamoxifen and placebo groups (31 and 26, respectively; $p=0.8$ ) and a nonsignificant excess of deaths in the tamoxifen $\operatorname{arm}(5.1 \%$ [182/3579] v. $4.6 \%$ [166/3575] in the placebo group; $p=0.4) .{ }^{10}$ These sobering findings occurred despite the persistent reduction in breast cancer incidence. 
At the same time, these drugs have clear harms. A recent trial that assigned more than 1000 premenopausal women to receive five years of adjuvant tamoxifen therapy reported high rates of adverse effects: ${ }^{11}$ more than $75 \%$ experienced hot flashes, at least $45 \%$ experienced depression, sweating and insomnia, and more than $40 \%$ experienced vaginal dryness and decreased libido. Hot flashes and depression were scored as grade 3 or 4 events (severe enough to limit a person's ability to care for themselves) in more than $7 \%$ and $3 \%$ of women, respectively. In a pooled analysis, ${ }^{9}$ both tamoxifen and raloxifene were found to increase the risk of venous thromboembolism (relative risk 1.93 and 1.60 , respectively). In addition, tamoxifen was associated with an increased rate of endometrial cancer (relative risk 2.13). ${ }^{9}$ All chemoprevention drugs appear to increase the risk of unpleasant vasomotor effects. In a recent placebo-controlled RCT of exemestane, the drug was associated with worse vasomotor symptoms, bodily pain and sexual domain scores. ${ }^{12}$ In another RCT, anastrozole was found to increase the risk of musculoskeletal effects, hypertension, vaginal dryness and vasomotor symptoms. ${ }^{13}$ Both aromatase inhibitors worsened bone mineral density. ${ }^{12,13}$ Because of such adverse effects, $25 \%-40 \%$ of trial participants stopped using the chemoprevention drugs. ${ }^{6}$

Discontinuation of drug use among the wellselected patients in clinical trials likely underestimates noncompliance in general clinical settings. The discontinuation of tamoxifen among young women receiving treatment for breast cancer (where a survival benefit is clear) was nearly $40 \%$ at three years in one communitybased study. ${ }^{14}$ Similar discontinuation rates were found for chemoprevention. Data from a highrisk breast clinic showed that $49.2 \%$ of 118 women stopped taking chemoprevention medication at least temporarily, largely because of adverse effects. ${ }^{15}$ The study also reported a high uptake of chemoprevention among those offered it $(54.4 \%)$; the rate exceeds that of many previous studies, which found uptake to fall between $10 \%$ and $15 \% .^{16}$

Despite these challenges, avoiding a cancer diagnosis appears an undisputed benefit. However, emerging appreciation of the rate of overdiagnosis from cancer screening programs adds additional complexity to this calculus. Incident cancer is considered the gold-standard outcome in chemoprevention trials, but prevention trials of some tumour types call this outcome into question. In 2013, the Prostate Cancer Prevention Trial found that, after 18 years of follow up, finasteride did not significantly improve overall survival compared with placebo $(78.0 \%$ and $78.2 \%$, respectively) in an RCT involving more than 18000 men. ${ }^{17}$ In addition, mortality among those found to have prostate cancer was similar in each study arm. An earlier report had shown that finasteride reduced incident prostate cancer, although it also suggested an increase in tumours with high Gleason scores. ${ }^{18}$ Whether or not these results were spurious was debated for years. It now seems clear that the cancers avoided by finasteride use do not contribute substantially to loss of life.

A similar phenomenon may be occurring with the agents used for chemoprevention of breast cancer. The reduction in incident cancer may preferentially occur among less aggressive breast tumours; thus, rates of fatal breast cancer may be unchanged. This hypothesis is supported by treatment data for ductal carcinoma in situ. A Cochrane meta-analysis found that tamoxifen decreased the incidence of future invasive breast cancer (ipsilateral: hazard ratio $0.79,95 \%$ confidence interval $[\mathrm{CI}]$ 0.62-1.01; contralateral: relative risk [RR] $0.57,95 \%$ CI $0.39-0.83$ ), but it did not change the risk of distant metastasis (RR 0.78, 95\% CI 0.29-2.08) or all-cause death (RR 1.11, 95\% CI 0.89-1.39) (breast cancer mortality was not reported). ${ }^{19}$

Some might argue that a breast cancer diagnosis should be avoided even if it proves not be fatal. The occurrence of breast cancer prompts further treatment, often including surgery, radiation and chemotherapy. Preventing a diagnosis of breast cancer avoids interventions with attendant costs, anxiety and morbidity. However, the absolute reduction in cancer incidence is likely smaller in the real world setting than in our analysis. The trials we examined were largely conducted in the 1990s, before recommendations were issued to reduce the frequency of breast cancer screening. Because of the phenomenon of overdiagnosis, recommendations to shift from annual to biannual mammography are expected to decrease the absolute incidence of invasive breast cancer. Accordingly, the absolute benefit of chemoprevention drugs will also decline. If the cancers prevented by these drugs are disproportionately overdiagnosed tumours (a hypothesis that cannot be contradicted from the available data), then it is unclear whether preventing a cancer that would not otherwise cause death or morbidity constitutes benefit. Some argue that, at a minimum, chemoprevention offers psychological value. However, the question of whether the net effect of chemoprevention - comparing the harms occurring in many individuals with the benefits in one - results in improved quality of life remains unanswered and unaddressed. 
Others believe that chemoprevention of breast cancer might work if done right. Perhaps a different drug, such as an aromatase inhibitor, with sufficient follow-up may someday show decreased cancer mortality. Although there are many reasons why trials have not yet shown a reduction in breast cancer mortality, such as insufficient power or follow-up, poor compliance, highly selected patient populations and the wrong drug, we must also entertain the possibility that the intervention does not improve this outcome.

Chemoprevention of breast cancer is not yet justified in women at increased risk of breast cancer. The medications have tangible adverse effects, and discontinuation rates are high. Moreover, the effects of treatment on the most important clinical outcome (death) remain uncertain, and the effects on incident cancer are likely overstated and contingent on high rates of screening. Asking a healthy patient to take a medication to prevent illness requires convincing evidence, particularly when adverse effects are common and severe. When it comes to the prevention of breast cancer, this bar has not yet been reached.

\section{References}

1. Moyer VA. Medications for risk reduction of primary breast cancer in women: US Preventive Services Task Force recommendation statement. Ann Intern Med 2013;159:698-708.

2. Visvanathan K, Hurley P, Bantug E, et al. Use of pharmacologic interventions for breast cancer risk reduction: American Society of Clinical Oncology clinical practice guideline. J Clin Oncol 2013; 31:2942-62.

3. Levine M, Moutquin JM, Walton R, et al. Chemoprevention of breast cancer: a joint guideline from the Canadian Task Force on Preventive Health Care and the Canadian Breast Cancer Initiative's Steering Committee on Clinical Practice Guidelines for the Care and Treatment of Breast Cancer. CMAJ 2001;164:1681-90.

4. Reducing your risk for breast cancer. Toronto: Canadian Cancer Society. Available: www.cancer.ca/en/cancer-information/cancer -type/breast/risks/reducing-your-risk/?region=on (accessed 2015 Mar. 17).

5. Freedman AN, Graubard BI, Rao SR, et al. Estimates of the number of US women who could benefit from tamoxifen for breast cancer chemoprevention. J Natl Cancer Inst 2003;95:526-32.
6. Cuzick J, Sestak I, Bonanni B, et al. Selective oestrogen receptor modulators in prevention of breast cancer: an updated meta-analysis of individual participant data. Lancet 2013;381:1827-34.

7. Goss PE, Ingle JN, Alés-Martínez JE, et al.; NCIC CTG MAP.3 Study Investigators. Exemestane for breast-cancer prevention in postmenopausal women. N Engl J Med 2011;364:2381-91.

8. King MC, Wieand S, Hale K, et al. Tamoxifen and breast cancer incidence among women with inherited mutations in BRCAl and BRCA2: National Surgical Adjuvant Breast and Bowel Project (NSABP-P1) Breast Cancer Prevention trial. JAMA 2001; 286:2251-6.

9. Nelson HD, Smith MEB, Griffin JC, et al. Use of medications to reduce risk for primary breast cancer: a systematic review for the US Preventive Services Task Force. Ann Intern Med 2013;158: 604-14.

10. Cuzick J, Sestak I, Cawthorn S, et al. Tamoxifen for prevention of breast cancer: extended long-term follow-up of the IBIS-I breast cancer prevention trial. Lancet Oncol 2015;16:67-75.

11. Francis PA, Regan MM, Fleming GF, et al. Adjuvant ovarian suppression in premenopausal breast cancer. N Engl J Med 2015; 372:436-46.

12. Maunsell E, Goss PE, Chlebowski RT, et al. Quality of life in MAP.3 (Mammary Prevention 3): a randomized, placebocontrolled trial evaluating exemestane for prevention of breast cancer. J Clin Oncol 2014;14:1427-36.

13. Cuzick J, Sestak I, Forbes JF, et al. Anastrozole for prevention of breast cancer in high-risk postmenopausal women (IBIS-II): an international, double-blind, randomised placebo-controlled trial. Lancet 2014;383:1041-8.

14. Huiart L, Bouhnik AD, Rey D, et al. Early discontinuation of tamoxifen intake in younger women with breast cancer: Is it time to rethink the way it is prescribed? Eur J Cancer 2012;48:1939-46.

15. Roetzheim RG, Lee JH, Fulp W, et al. Acceptance and adherence to chemoprevention among women at increased risk of breast cancer. Breast 2015;24:51-6.

16. Donnelly LS, Evans DG, Wiseman J, et al. Uptake of tamoxifen in consecutive premenopausal women under surveillance in a high-risk breast cancer clinic. Br J Cancer 2014;110:1681-7.

17. Thompson IM, Goodman PJ, Tangen CM, et al. Long-term survival of participants in the Prostate Cancer Prevention Trial. N Engl J Med 2013;369:603-10.

18. Thompson IM, Goodman PJ, Tangen CM, et al. The influence of finasteride on the development of prostate cancer. $N$ Engl J Med 2003;349:215-24.

19. Staley H, McCallum I, Bruce J. Postoperative tamoxifen for ductal carcinoma in situ. Cochrane Database Syst Rev 2012; (10):CD007847.

Affiliations: Bloomberg School of Public Health (Prasad); Department of Biostatistics (Diener-West), Bloomberg School of Public Health, Johns Hopkins University, Baltimore, Md.

Contributors: Both authors contributed substantially to the drafting and revising of the manuscript, approved the final version submitted for publication and agreed to act as guarantors of the work. 